Working Paper : \#09-37 (14)

Statistics and Econometrics Series

June 2009
Departamento de Estadística

Universidad Carlos III de Madrid

Calle Madrid, 126

28903 Getafe (Spain)

Fax (34) 91 624-98-49

\title{
CONTROLLED DIFFUSION PROCESSES WITH
} MARKOVIAN SWITCHINGS FOR MODELING DYNAMICAL ENGINEERING SYSTEMS

\author{
Héctor Cañada* and Rosario Romera**. \\ * Department of Statistics, Universidad Carlos III de Madrid, Av/ Universidad no \\ 30, 28911 Leganés (Madrid), Spain \\ ** Department of Statistics, Universidad Carlos III de Madrid, C/ Madrid $\mathbf{n}^{\circ}$ 126; \\ 28903 Getafe (Madrid), Spain. \\ e-mail: jaimehector.canada@uc3m.es \\ rosario.romera@uc3m.es
}

\begin{abstract}
A modeling approach to treat noisy engineering systems is presented. We deal with controlled systems that evolve in a continuous-time over finite time intervals, but also in continuous interaction with environments of intrinsic variability. We face the complexity of these systems by introducing a methodology based on Stochastic Differential Equations (SDE) models. We focus on specific type of complexity derived from unpredictable abrupt and/or structural changes. In this paper an approach based on controlled Stochastic Differential Equations with Markovian Switchings (SDEMS) is proposed. Technical conditions for the existence and uniqueness of the solution of these models are provided. We treat with nonlinear SDEMS that does not have closed solutions. Then, a numerical approximation to the exact solution based on the EulerMaruyama Method (EM) is proposed. Convergence in strong sense and stability are provided. Promising applications for selected industrial biochemical systems are showed.
\end{abstract}

Keywords: Markov chains, stochastic dynamical systems, numerical approaches for $S D E$. 


\title{
Controlled Diffusion Processes with Markovian Switchings for Modeling Dynamical Engineering Systems.
}

\author{
Héctor Cañada* and Rosario Romera. \\ Departamento de Estadística. Universidad Carlos III de Madrid.
}

June 29, 2009

\begin{abstract}
Dynamical engineering systems are controlled systems that evolve in continuous time over finite time intervals, but also in continuous interaction with environments of intrinsic variability. In this paper, the complexity of these systems is treated by introducing an approach based on controlled Stochastic Differential Equations with Markovian Switchings $(S D E M S)$. Technical conditions for the existence and uniqueness of solution for controlled $S D E M S$ equations are provided. The rule and not the exception is to deal with nonlinear SDEMS that doesn't have closed solutions. Then, a numerical approximation to the exact solutions based on the Euler-Maruyama (EM) method is proposed. Convergence in strong sense and stability are provided. Promising applications for selected industrial biochemical systems are showed.

Keywords:Markov chains, stochastic dynamical systems, numerical approaches for $S D E$.

* Corresponding author : jaimehector.canada@uc3m.es. Av. Universidad 30, 28911, Leganés, Madrid. Spain.

Acknowledgements: The authors would like to thanks the support research provided by the Spanish Ministry of Education and Science under project $2007 / 04438 / 001$.
\end{abstract}

\section{Introduction}

System of relevant importance in many branches of engineering are controlled dynamical systems (in what follows engineering systems) that evolve in continuous time over finite time intervals, which are also in constant interaction with their environments. In industrial scenarios, the system environments have a high degree of heterogeneity. In other words, they are intrinsically "noisy". Even though a wide class of this noise is spurious, with null effects in the dynamics of the system, does exist other classes of variability that affects the behavior of the 
systems in a complex and unpredictable way, for example the "structural noise". Furthermore, in engineering systems, as a result of the medium heterogeneity and the system-environment interactions, frequently emerge a more complex type of variability that can be considered as an "hybrid noisy dynamics". That dynamics is a characteristic feature of engineering system that have its origin when the evolution of the system (in a continuous-time) is modified for unpredictable changes governed by discrete events such as changes in the physical medium properties, the operation conditions; control decisions, the presence of regimes, the interaction with other engineering systems, among others. Events of this nature are associated with abrupt and/or structural changes in the dynamics of the system. In summary, engineering systems are complex systems that requires more sophisticated tools of description and analysis beyond the scope of deterministic models. More realistic models for the description of engineering systems allow us for example, to design better control protocols during its operation.

Two examples allow us to illustrate the ideas previously discussed. The first one is related to the extraction equipments used in the petroleum industry. The dynamics of these equipments depends on the underground oil flow. The fluid dynamics is governed by diffusion phenomena (connected with diffusion parameters in the models) that are sensible to changes in the properties of the porous medium or the chemical properties of the fluids. The complexity and unpredictablility of these changes makes very difficult to have a realistic description of the diffusion dynamics. In consequence, the control operation of the extraction equipment would be very limited at least noise effects would be taken into account. The second one is concerned about biochemical industrial reactors (bioreactors) used to produce pharmaceuticals. The bioreactors are systems extremely sensible to environmental fluctuations (temperature, $\mathrm{pH}$, concentrations of nutrients, etc.) because of the sensibility of the microorganisms that carry out the chemical transformations from the initial material to the final product (connected with kinetic parameters in the models). The persistency of that fluctuations may produce microorganisms mutations that perturb the chemical routes to the final product with the consequent changes of regimes in the dynamics of the system. If that fluctuations are not considered it is not possible to take the correct control decisions according with the regime that govern the dynamics of the system.

This paper is concerned with modelling noisy engineering system typical of industrial scenarios. We face that problem by consider them as Stochastic Dynamical Systems (see: [1]), in particular systems governed by Stochastic Differential Equations (SDE). We consider an approach based on Stochastic Differential Equations with Markovian Switchings (SDEMS) (see: [15]). The resulting models are named controlled SDEMS. Our work offer a systematic treatment with emphasis in structural and abrupt noise. This approach allow us to treat noisy aspects, but also the hybrid nature of these systems by incorporating Markov chains to model discret-time events embedded in the continuous-time dynamics of the system. With this approach we offer a more complete description of engineering systems that is not possible using the classics $S D E$ models 
(see for example: [12] and [18]). Even though this problem has been studied in the context of the hybrid systems area, where does exist vast literature in theory and applications topics (see: [6]), our treatment is more suitable in the context of the $S D E$. The advantage of the $S D E$ approach is that allow us to preserve the intrinsic properties of the systems under analysis, but at the same time to introduce the stochastic process elements that deal with the uncertainty.

The SDEMS models was first treated in the $S D E$ area in [15], and has been applied successfully in economy an finance (see for example: [3]). Nowadays, as far as we know, does not exist applications developed in engineering areas, with the exception of [8], which is focused in the manufacturing systems control area. Furthermore, in engineering the rule and not the exception is to deal with nonlinear systems, but also with manipulable (controllable) systems. With respect to the first point, that means that we deal with nonlinear SDEMS. Then, we need to implement numerical methods of approximation to the exact solutions. In this paper, we propose an approach based on the original EulerMaruyama (EM) method (see: [16] and [10]). Aspects related to convergence in strong sense and local stability are considered to guarantee a well defined and reliable approximated solution. The second point is detailed as follows.

Most of the engineering systems are driven by external agents, through inputaffine variables to the system (input parameters in the models). For one side we have control variables, related with "intelligent" controllers that act over the systems with a given protocol. . For the other side there exists exogenous variables (environmental variables), related with the "uncontrollable" and unpredictable complex system environment. We remark that this paper is not devoted to find optimal strategies of control, but our results may be easily translated to the optimization context. The model proposed in this paper to incorporate the control variables to the original $S D E M S$ models. As a result we have controlled SDEMS models for which technical conditions of existence and uniqueness of the solutions are provided.

On the other hand, we provide of dynamics to the physical system parameters that usually are considered fixed in many applications. Notice that these parameters play a fundamental role in modeling because they concentrate relevant information about the physical laws that govern the system like those concerning thermodynamics, equilibrium, among others. In a few words, physical system parameters work as sensors of change into the fundamental dynamics of the system. These parameters will be referred as structural parameters in our model.

The main contribution of this paper is to provide an unified framework to model engineering systems for which different sources of variability (intrinsic and external) are considered as well as the effect of external controllers. We provide mathematical and numerical results that let us to obtain numerical solutions for dynamical systems that evolve as controlled SDEMS.

Finally, we highlight the potential utility of the controlled SDEMS models trough an application in the biochemical engineering area.

This paper is organized as follows: Section 2 introduces the model approach based in controlled SDEMS models and include proofs for the existence 
and uniqueness conditions of the solution. Section 3 describes the numerical approximation scheme based in the EM-based method. Section 3 shows an application of our model to the biochemical engineering area. Section 4 summarizes conclusions and future lines of research.

\section{The model and the mathematical support}

We model a noisy engineering system as a set of controlled SDEMS denoted as follows

$$
d x(t)=f\left(x(t), u_{t}, r(t)\right) d t+g\left(x(t), u_{t}, r(t)\right) d B_{t} ; \quad t \in\left[t_{0}, T\right]
$$

where $x_{t}$ is the state variable taking values in $\mathbb{R}^{n} ; u_{t}=u(\cdot)$ is a given control protocol taking values in a compact set $U \subset \mathbb{R}$ that may depends on the state of the system and other elements; $r(t)$ is a continuous time Markov chain that governs discrete events in the dynamics of the system; $\left\{B_{t}, t \geq 0\right\}$ is a standard Brownian motion taking values in $\mathbb{R}^{m}$.

The dynamics of the system is characterized by the drift function, $f: \mathbb{R}^{n} \times$ $U \times S \rightarrow \mathbb{R}^{n}$ and the diffusion function, $g: \mathbb{R}^{n} \times U \times S \rightarrow \mathbb{R}^{n \times m}$.

With more detail, discrete events are driven by a stochastic process $r(\cdot) \equiv$ $\{r(t), t \geq 0\}$, here assumed a right continuous-time homogeneous Markov chain on the probability space $(\Omega, \mathcal{F}, P)$, with values in a finite state space $S=$ $\{1,2, \cdots, N\}$ and with generator $\Gamma=\left(\gamma_{i j}\right)_{N \times N}$ given by

$$
P\left\{r(t+\Delta=j \mid r(t)=i\}=\left\{\begin{array}{c}
\gamma_{i j} \Delta+o(\Delta) \text { if } i \neq j \\
1+\gamma_{i i} \Delta+o(\Delta) \text { if } i=j
\end{array}\right\}\right.
$$

where $\Delta>0$. Here $\gamma_{i j} \geq 0$ is the transition rate from $i$ to $j$ if $i \neq j$ while

$$
\gamma_{i i}=-\sum_{j \neq i} \gamma_{i j}
$$

We assume that the Markov chain $r(\cdot)$ is $\mathcal{F}_{t}$-adapted and independent of the Brownian motion $B(\cdot)$.

Notice that (1) can be regarded as a system of $N$ equations

$$
d x(t)=f\left(x(t), u_{t}, i\right) d t+g\left(x(t), u_{t}, i\right) d B_{t} ; \quad 1 \leq i \leq N .
$$

switching from one to another state $i$ according to the evolution of the Markov chain $r(\cdot)$. Notice also that (1) is an autonomous system (not depends explicitly on time).

A process $\{x(t)\}_{t_{0} \leq t \leq T}$ that satisfies (1) is a stochastic process that is said to be $\mathcal{F}_{t}$-adapted if for every $t, x(t)$ is $\mathcal{F}_{t}$-measurable. With this assumption we provide the following definition. 
Definition 1 Given an control protocol $u(\cdot)$ in $\left[t_{0}, T\right]$, a $\mathbb{R}^{n}$-valued stochastic process $\{x(t)\}_{t_{0} \leq t \geq T}$ is called a solution of the equation (1) if it has the following properties

$$
\{x(t)\}_{t_{0} \leq t \leq T}
$$

is continuous and $\mathcal{F}_{t}$-adapted.

$$
\begin{gathered}
f\left(x(t), u_{t}, r(t)\right)_{t_{0} \leq t \leq T} \in \mathcal{L}^{1}\left(\left[t_{0}, T\right] ; \mathbb{R}^{n}\right) \\
\left\{g\left(x(t), u_{t}, r(t)\right)\right\}_{t_{0} \leq t \leq T} \in \mathcal{L}^{2}\left(\left[t_{0}, T\right] ; \mathbb{R}^{n \times m}\right)
\end{gathered}
$$

the equation

$$
x(t)=x\left(t_{0}\right)+\int_{t_{0}}^{t} f\left(x(s), u_{s}, r(s)\right) d s+\int_{t_{0}}^{t} g\left(x(s), u_{s}, r(s)\right) d B_{s}
$$

holds with probability 1. A solution $\{x(t)\}_{t_{0} \leq t \leq T}$ is said to be unique if any other solution $\{\bar{x}(t)\}_{t_{0} \leq t \leq T}$ is indistinguishable from the former. To ensure the uniqueness of the solution we need to guarantee some conditions with respect to the functions $f, g$ and the Markov chain as we will show in Theorem 1.

Definition 2 Every sample path of $r(\cdot)$ is a right-continuous step function with a finite number of simple jumps on $\left[t_{0}, T\right]$. So there is a sequence $\left\{\tau_{k}\right\}_{k \geq 0}$ of stopping times such that (i) for almost every $\omega \in \Omega$ there is a finite $\bar{k}=\bar{k}(\omega)$ for $t_{0}=\tau_{0}<\tau_{1}<\cdots<\tau_{\bar{k}}=T$ and $\tau_{\bar{k}}=T$ if $k>\bar{k}$ (ii) $r(\cdot)$ is a random constant where $r(t)=r\left(\tau_{k}\right)$ on $\tau_{k} \leq t<\tau_{k+1}$.

Theorem 1 Assume that functions $f(x, u, i)$ and $g(x, u, i)$ from Definition 1 are measurable and satisfies, for some positive constants $\bar{K}$ and $K$, the following properties

(a) (Lipschitz condition) for all $x, y \in \mathbb{R}^{n}, u \in U$ and $i \in S$

$$
|f(x, u, i)-f(y, u, i)|+|g(x, u, i)-g(y, u, i)| \leq \bar{K}|x-y|
$$

(b) (Linear growth condition) for all $(x, u, i) \in \mathbb{R}^{n} \times U \times S$

$$
|f(x, u, i)|^{2}+|g(x, u, i)|^{2} \leq K\left(1+|x|^{2}\right)
$$

In addition, the Markov chain $r(\cdot)$ is such that the joint process $(x(\cdot), r(\cdot))$ satisfies the Feller property. Then, there is a solution $x(t)$ of the equation (1) and, moreover, this is unique.

Proof. The main ideas of this proof are borrowed from [2] and [15]. First, with the aid of the Picard iterative scheme, we will prove existence; after that we 
will prove uniqueness. We start considering (1) on the interval $\left[\tau_{0}, \tau_{1}\right]=\left[t_{0}, \tau_{1}\right]$ (From Definition 2). It yields

$$
d x(t)=f\left(x(t), u_{t}, r_{0}\right) d t+g\left(x(t), u_{t}, r_{0}\right) d B_{t}
$$

Existence: In engineering systems, the initial conditions $x\left(t_{0}\right)=x_{0}$ are bounded by physical constraints, so we have the case

$$
E\left[\left|x_{0}\right|^{2}\right] \leq \infty
$$

Let us begin an iterative procedure with $x\left(t_{0}\right)=x_{0}$ and let us define, for $n \geq 1$, $t \in\left[t_{0}, \tau_{1}\right]$ and a control protocol $u_{t}$ on the same time interval

$$
x_{n}(t)=x_{0}+\int_{t_{0}}^{t} f\left(x_{n-1}(s), u_{s}^{n-1}, r_{0}\right) d s+\int_{t_{0}}^{t} g\left(x_{n-1}(s), u_{s}^{n-1}, r_{0}\right) d B_{s}
$$

By virtue of the inequality $|a+b+c|^{2} \leq 3\left(|a|^{2}+|b|^{2}+|c|^{2}\right)$ and linear growth condition (b), it follows from (6) that

$$
E\left[\left|x_{n}(t)\right|^{2}\right] \leq K_{1}\left(1+\sup _{t_{0} \leq t \leq \tau_{1}} E\left[\left|x_{n-1}(t)\right|^{2}\right]\right)
$$

so it yields

$$
E\left[\left|x_{n}(t)\right|^{2}\right] \leq \infty
$$

Notice that this is true for $n=t_{0}$ as was discussed above. Now, the idea is to show that $x_{n}(t)$ converges uniformly on $\left[t_{0}, \tau_{1}\right]$ to a solution $x(t)$ of $(5)$. To do that, let us define

$$
\begin{aligned}
z_{n+1}(t)= & \int_{t_{0}}^{t}\left[f\left(x_{n}(s), u_{s}, r_{0}\right)-f\left(x_{n-1}(s), u_{s}, r_{0}\right)\right] d s+ \\
& +\int_{t_{0}}^{t} g\left(x_{n}(s), u_{s}, r_{0}\right)-g\left(x_{n-1}(s), u_{s}, r_{0}\right) d B_{s}
\end{aligned}
$$

By virtue of the inequality $|a+b|^{2} \leq 2\left(|a|^{2}+|b|^{2}\right)$, Shwarz's inequality, integral stochastic properties and Lipschitz condition

$$
E\left[\left|z_{n+1}(t)\right|^{2}\right] \leq L\left[\int_{t_{0}}^{t} E\left|z_{n}(t)\right|^{2} d s\right]
$$

with $L=2\left(\tau_{1}-t_{0}+1\right) \bar{K}^{2}$. For iteration on this inequality, using the Cauchy formula we get

$$
E\left[\left|z_{n+1}(t)\right|^{2}\right] \leq L^{n} \int_{t_{0}}^{t} \frac{(t-s)^{-1}}{(n-1) !} E\left|z_{2}(t)\right|^{2} d s
$$

Now, under the assumption the linear growth condition (b),

$$
E\left|Z_{2}(t)\right|^{2}=E\left|x_{1}(t)-x_{0}(t)\right|^{2} \leq L\left(\tau_{1}-t_{0}\right)\left(1+E\left[\left|x_{0}\right|^{2}\right]\right)
$$


then

$$
\sup _{t_{0} \leq t \leq \tau_{1}} E\left[\left|z_{n+1}(t)\right|^{2}\right] \leq \frac{\left[C\left(L\left(\tau_{1}-t_{0}\right)\right)^{n}\right]}{n !}, \quad n \geq 0
$$

by using (6) we have that

$$
\begin{aligned}
\sup _{t_{0} \leq t \leq \tau_{1}}\left|z_{n+1}(t)\right| \leq & \int_{t_{0}}^{t}\left|f\left(x_{n}(s), u_{s}^{n}, r_{0}\right)-f\left(x_{n-1}(s), u_{s}^{n-1}, r_{0}\right)\right| d s \\
& +\sup _{t_{0} \leq t \leq \tau_{1}}\left|\int_{t_{0}}^{t}\left[g\left(x_{n}(s), u_{s}^{n}, r_{0}\right)-g\left(x_{n-1}(s), u_{s}^{n-1}, r_{0}\right)\right] d B_{s}\right|
\end{aligned}
$$

If we use the Ito's integral properties, inequality $|a+b|^{2} \leq 2\left(|a|^{2}+|b|^{2}\right)$ and Lipschitz condition (a) we get

$$
\begin{aligned}
E\left[\sup _{t_{0} \leq t \leq \tau_{1}}\left|z_{n+1}(t)\right|^{2}\right] \leq & 2\left(\tau_{1}-t_{0}\right) \bar{K}^{2} \int_{t_{0}}^{t} E\left|x_{n}(s)-x_{n-1}(s)\right|^{2} d s \\
& +8 \bar{K}^{2} \int_{t_{0}}^{t} E\left|x_{n}(s)-x_{n-1}(s)\right|^{2} d s
\end{aligned}
$$

this result in addition with (7) yields

$$
E\left[\sup _{0 \leq t \leq \tau_{1}}\left|z_{n+1}(t)\right|^{2}\right] \leq \frac{\left[C_{1}\left(L\left(\tau_{1}-t_{0}\right)\right)^{n-1}\right]}{n-1 !}, \quad n \geq 0
$$

Since $\sum_{n=1}^{\infty} \frac{\left[C_{1}\left(L\left(\tau_{1}-t_{0}\right)\right)^{n-1}\right]}{n-1 !} \leq \infty$, the Borel-Cantelli lemma and the Weierstrass's criteria ensure convergence of the series

$$
\sum_{n=1}^{\infty} P\left[\sup _{t_{0} \leq t \leq \tau_{1}}\left|z_{n+1}(t)\right|>n^{-2}\right] \leq C_{1} \sum_{n=1}^{\infty} \frac{\left[\left(L\left(\tau_{1}-t_{0}\right)\right)^{n-1} n^{4}\right]}{n-1 !}
$$

which implies that, the limit expression

$$
\lim _{n \rightarrow \infty}\left(x_{0}(t)+\sum_{i=1}^{n}\left(x_{i}(t)-x_{i-1}(t)\right)\right)=\lim _{n \rightarrow \infty} x_{n}(t)=x(t)
$$

holds with probability 1 , uniformly in $\left[t_{0}, \tau_{1}\right]$. Since $x(t)$ is the limit of a sequence of nonaticipating-functions and the uniform limit of a sequence of continuous functions, it is itself a nonanticipating and continuous. The right hand member of (5) becomes a $S D E$, when we replace $x(t)$ into it. But we need to show that $x(t)$ satisfy the equation

$$
x(t)=x_{0}+\int_{t_{0}}^{t} f\left(x(s), u_{s}, r_{0}\right) d s+\int_{t_{0}}^{t} g\left(x(s), u_{s}, r_{0}\right) d B_{s}
$$

for all $t \in\left[t_{0}, \tau_{1}\right]$. Notice that it is true for $t=t_{0}$, because $x_{n}\left(t_{0}\right)=x_{0}$, (for $n \geq 0)$. Now we need to prove that for $\left(t_{0}, \tau_{1}\right]$. From (6), (5) and the Lipschitz 
condition (b) we get

$$
\begin{aligned}
\int_{t_{0}}^{t}\left|f\left(x_{n}(s), u_{s}^{n}, r_{0}\right)-f\left(x(s), u_{s}, r_{0}\right)\right| d s & \leq \int_{t_{0}}^{t} \bar{K}\left|x_{n}(s)-x(s)\right| d s \\
\int_{t_{0}}^{t}\left|g\left(x_{n}(s), u_{s}^{n}, r_{0}\right)-g\left(x(s), u_{s}, r_{0}\right)\right| d B_{s} & \leq \int_{t_{0}}^{t} \bar{K}\left|x_{n}(s)-x(s)\right| d B_{s}
\end{aligned}
$$

By takin limit in the above expression we can conclude that, with probability 1

$$
\lim _{n \rightarrow \infty} \int_{t_{0}}^{t} f\left(x_{n}(s), u_{s}^{n}, r_{0}\right) d s=\int_{t_{0}}^{t} f\left(x(s), u_{s}, r_{0}\right) d s
$$

and

$$
\lim _{n \rightarrow \infty} \int_{t_{0}}^{t} f\left(x_{n}(s), u_{s}^{n}, r_{0}\right) d s=\int_{t_{0}}^{t} f\left(x(s), u_{s}, r_{0}\right) d B_{s}
$$

holds. Therefore, $x(t)$ is solution of (8.). If we repeat this procedure up to the time interval $\left[\tau_{k}, T\right]$ the proof of existence is completed.

Uniqueness: Let $x(t)$ and $\bar{x}(t)$ be two solutions of equation (5). We need to show that

$$
E|x(t)-\bar{x}(t)|=0 \quad \forall t \in\left[t_{0}, \tau_{1}\right]
$$

from (5) we have

$$
\begin{aligned}
x(t)-\bar{x}(t)= & \int_{t_{0}}^{t}\left[f\left(x(s), u_{s}, r_{0}\right)-f\left(\bar{x}(s), u_{s}, r_{0}\right)\right] d s+ \\
& +\int_{t_{0}}^{t} g\left(x(s), u_{s}, r_{0}\right)-g\left(\bar{x}(s), u_{s}, r_{0}\right) d B_{s}
\end{aligned}
$$

for $s \in\left[t_{0}, t\right]$ By virtue of Lipschitz condition (a) implies

$$
\begin{aligned}
& \left|f\left(x(s), u_{s}, r_{0}\right)-f\left(\bar{x}(s), u_{s}, r_{0}\right)\right|+\left|g\left(x(s), u_{s}, r_{0}\right)-g\left(\bar{x}(s), u_{s}, r_{0}\right)\right| \\
\leq & 2 \bar{K}|x(s)-\bar{x}(s)|
\end{aligned}
$$

which ensure the existence of the second moments of both integrals in (9). Now, by virtue of the inequality $|a+b|^{2} \leq 2\left(|a|^{2}+|b|^{2}\right)$ and the Ito's integral properties

$$
\begin{aligned}
E\left[|x(t)-\bar{x}(t)|^{2}\right] \leq & 2\left(\tau_{1}-t_{0}\right) \int_{t_{0}}^{t} E\left[\left|f\left(x(s), u_{s}, r_{0}\right)-f\left(\bar{x}(s), u_{s}, r_{0}\right)\right|^{2}\right] d s+ \\
& \int_{t_{0}}^{t}\left[\left|g\left(x(s), u_{s}, r_{0}\right)-g\left(\bar{x}(s), u_{s}, r_{0}\right)\right|^{2}\right] d s
\end{aligned}
$$

From Lipschitz condition (a) we get

$$
E\left[|x(t)-\bar{x}(t)|^{2}\right] \leq L \int_{t_{0}}^{t} E\left[|x(s)-\bar{x}(s)|^{2}\right] d s
$$


with $L=2\left(\tau_{1}-t_{0}+1\right) \bar{K}^{2}$. By virtue of the Gronwall inequality we conclude that

$$
E\left[|x(t)-\bar{x}(t)|^{2}\right]=0 \quad \forall t \in\left[t_{0}, \tau_{1}\right]
$$

By applying of this procedure up to the time interval $\left[\tau_{k}, T\right]$ the proof is completed.

Remark 1 The Lipschitz condition (a) from Theorem 1 does ensure that the realizations of the stochastic process $x(t)$ (the solution of (1)) does not diverge, that is, the state variable does not go to infinity in a finite time interval. The linear growth condition (b) from Theorem 1 allows at most linear increase of function $f$ and $g$ in (1) with respect to $x$ and guarantee that, with probability 1 , the solution $x(t)$ does not explode in the interval $t \in\left[t_{0}, T\right]$ for a given initial condition $x_{0}$. In practice, to verify Lipschitz conditions for the equation (1) could be troublesome. Fortunately a convenient sufficient condition comes from the mean-value theorem of differential calculus (see [1]). It is important to say that these conditions are very restrictive. Many system of practical interest in engineering does not verify these conditions. This problem is attempted by relaxing the cited conditions or ensuring a sufficient regularity in the functions (Drift and Diffusion functions) that govern the dynamics of the system. Remember that given conditions are sufficient but not necessary, so there are others ways to guarantee that the solution of the problems we face are tractable. Fortunately most of the equations that govern engineering systems are continuous and derivable with sufficient regularity to ensure a well defined solution.

\section{Numerical approximation}

Most of the noisy engineering system are nonlinear. In consequence, we treat with nonlinear controlled SDEMS that does not have closed solutions. Under this scenario it is necessary to develop numerical approximations to the exact solutions. We are interested in pathwise approximations, because we need to describe the engineering systems through direct simulation. Many explicit and implicit methods based on discrete time approximations (see [12]) has been successfully implemented in many application of the $S D E$ models (see [21]). Recently, promising numerical approaches, based on the EM-method has been proposed to treat with SDEMS models (see for instance [16]). Unfortunately, as we will see later, this method result inappropriate for dealing with problems of practical interest as in our case. Nevertheless, our proposal shares the sake basis. i.e., the EM- method. Even tough this scheme have limitations related to numerical efficiency and accuracy for treating high nonlinear problems, in weak and moderate nonlinear problems it give us valuable information about the dynamics of the system. Our method is fast and relatively easy to implement, but also offer numerical local stability which is a very important property, because guarantee that the method is not explosive or diverge on a given finite time interval $\left[t_{0}, T\right]$ depending on the stepsize. Summarizing, in this section we 
detail our numerical approximation based on the EM-method as well as brief comparative analysis with the original method proposed in (see [16]).

We propose a numerical approximation for the diffusion process $\{x(t)\}_{t_{0} \leq t \leq T}$ (1) based on the EM-method. The method proposed is constructed as follows

For a given discretization $t_{0}=\tau_{0}<\tau_{1}<\cdots<\tau_{k}<\cdots<\tau_{N}=T$ of the time interval $\left[t_{0}, T\right]$, an Euler-Maruyama approximation is a continuous time stochastic process $X=\left\{X(t), t_{0} \leq t \leq T\right\}$ satisfying the iterative scheme

$X_{k+1}=I_{i, j}\left(r_{k+1}^{\Delta}\right)+f\left(I_{i, j}\left(r_{k+1}^{\Delta}\right), u_{k}, r_{k}^{\Delta}\right) \Delta+g\left(I_{i, j}\left(r_{k+1}^{\Delta}\right), u_{k}, r_{k}^{\Delta}\right) \Delta w_{k}$, for $k=1,2, . ., N-1$

where

$$
I_{i, j}\left(r_{k+1}^{\Delta}\right)=\left\{\begin{array}{ll}
X_{k}^{i} & \text { if } r_{k+1}^{\Delta}=r_{k}^{\Delta} \\
X_{k}^{j} & \text { if } r_{k+1}^{\Delta}=r_{k}^{\Delta}
\end{array}\right\}
$$

is the indicator function $I_{i, j}$, with $i \in S$, being the state of the Markov chain at the $k$-th step, $j \in S-1$, being the new state of the Markov chain at the $k+1$-th step and $X_{k}=X\left(\tau_{k}\right)$ the approximation at the discretization time $\tau_{k}$. Let $X_{0}=x_{0}, r_{0}^{\Delta}=r_{0}$ be the initial values of the state variable and the Markov chain respectively.

We consider $\Delta_{k}=\tau_{k+1}-\tau_{k}$ for the $k$-th time increment and call $\delta=\max _{k} \Delta_{k}$ the maximum time step, at this case assumed constant $\left(\Delta_{k}=\Delta\right)$. We define $\left\{r_{k}^{\Delta}=r(k \Delta)\right\}$ as a time discrete-time Markov chain with the one-step transition probability matrix $P(\Delta)=\left(P_{i j}(\Delta)\right)_{S \times S}=e^{\Delta \Gamma}$. (For details abut the Markov chain computing see [16]). Also we define $u_{k}=u\left(\tau_{k}\right)$ as the control protocol at the step $k$ in the time discretization.

Remark 2 In this work we shall consider equidistant discretization times $\tau_{k}=$ $\tau_{0}+\Delta$ with $\Delta=\frac{\left(T-t_{0}\right)}{N}$ for some integer $N$ large enough so that $\Delta \in(0,1)$.

\subsection{Strong convergence}

Because we need to extract information about the dynamics of the system through direct simulations with our models, we need to guarantee that our numerical approach converges in strong sense to the exact solution. In other words, we adopt the criteria that a discrete-time approximation $X^{\delta}$ with maximum step size $\delta$ converges to $x$ at time $T$ if

$$
\lim _{\delta \rightarrow 0} E\left[\left|x(T)-X^{\delta}(T)\right|\right]=0
$$

We show this property through the following result.

Theorem 2 If we assume (3) and (4) in Theorem 1, for a given control protocol 
$u(\cdot)$ in $\left[t_{0}, T\right]$ and $x, y \in \mathbb{R}^{n}$. In addition

$$
\begin{aligned}
\text { 1. } & E\left(\left|X_{0}\right|^{2}\right) & <\infty \\
\text { 2. } & E\left(\left|x_{0}-X_{0}\right|^{2}\right)^{1 / 2} & \leq K_{1} \delta^{1 / 2} \\
\text { 3. } & \delta & =\max \Delta_{k}=\Delta \\
\text { 4. } & r_{0}^{\Delta} & =r_{0}
\end{aligned}
$$

where $K_{1}$ is a positive constant that does not depends on $\delta$. Then for the EM approximation $X^{\delta}$ we have that

$$
E\left(\left|x(T)-X^{\delta}\right|\right) \leq \bar{K} \delta^{1 / 2}
$$

In reality this condition must holds for any time $t \in\left[t_{0}, T\right]$ but even more, according with the evolution of the Markov chain we need to verify this condition for the random partition of the finite time interval according with Definition 2. Notice that the expressions (10) and (11) are equivalent at the limit condition $\delta \rightarrow 0$

Proof. We first consider the evolution of system (1) on $\tau \in\left[\tau_{0}, \tau_{1}\right]$, with $\tau_{0}=$ $t_{0}$. If we define $Z(t)=E\left[\sup _{t_{0} \leq t \leq \tau_{1}}\left|x_{t}-X^{\delta}(t)\right|^{2}\right]$, by using the methodology proposed in [12] and [16] we get the following result

$$
Z(t) \leq C_{1}\left|x_{0}-X_{0}^{\delta}\right|^{2}+C_{2}\left(1+\left|x_{0}\right|^{2}\right) \delta+C_{3} \int_{t_{0}}^{t} Z(v) d v
$$

Now, by applying the Gronwall inequality

$$
Z(t) \leq \exp \int_{t_{0}}^{t} d v\left\{C_{3}\left|x_{0}-X_{0}^{\delta}\right|^{2}+C_{4}\left(1+\left|x_{0}\right|^{2}\right) \delta\right\}
$$

In $t=\tau_{1}$ we have

$$
E\left[\sup _{t_{0} \leq t \leq \tau_{1}}\left|x_{t}-X^{\delta}(t)\right|^{2}\right]=E\left[\left|x_{\tau 1}-X^{\delta}\left(\tau_{1}\right)\right|^{2}\right]
$$

then, from (12)

$$
E\left[\left|x_{\tau_{1}}-X^{\delta}\left(\tau_{1}\right)\right|^{2}\right] \leq C_{5}\left|x_{0}-X_{0}^{\delta}\right|^{2}+C_{6}\left(1+\left|x_{0}\right|^{2}\right) \delta
$$

taking the expected value

$$
E\left[\left|x_{\tau_{1}}-X^{\delta}\left(\tau_{1}\right)\right|^{2}\right] \leq C_{5} E\left[\left|x_{0}-X_{0}^{\delta}\right|^{2}\right]+C_{6}\left(1+\left|x_{0}\right|^{2}\right) \delta
$$

the square root yields

$$
E\left[\left|x_{\tau_{1}}-X^{\delta}\left(\tau_{1}\right)\right|^{2}\right]^{1 / 2} \leq C_{7} E\left[\left|x_{0}-X_{0}^{\delta}\right|^{2}\right]^{1 / 2}+C_{8}\left(1+\left|x_{0}\right|^{2}\right) \delta^{1 / 2}
$$


from the hypothesis (2)

$$
C_{7} E\left[\left|x_{0}-X_{0}^{\delta}\right|^{2}\right]^{1 / 2}+C_{8}\left(1+\left|x_{0}\right|^{2}\right) \delta^{1 / 2} \leq K_{1} \delta^{1 / 2}+C_{8}\left(1+\left|x_{0}\right|^{2}\right) \delta^{1 / 2}
$$

therefore

$$
E\left[\left|x_{\tau_{1}}-X^{\delta}\left(\tau_{1}\right)\right|^{2}\right]^{1 / 2} \leq K_{2} \delta^{1 / 2}
$$

By virtue of the Jensen inequality we conclude that

$$
E\left[\left|x_{\tau_{1}}-X^{\delta}\left(\tau_{1}\right)\right|\right] \leq K_{3} \delta^{1 / 2}
$$

Note that constants $C 1$ to $C 8$ and $K_{1}$ to $K_{3}$ are positive constants. If we repeat this procedure until $\tau_{k}=T$, the proof will be completed.

\subsection{Numerical stability}

We say, from an intuitive point of view, that a method is numerically stable in a finite interval $\left[t_{0}, T\right]$ if the propagation of an initial error of approximation remains bounded on that interval, which means that the intrinsic structure of the method avoids the growth of the error over the total time interval of interest. We formalize this property through the following result.

Theorem 3 Let $X^{\delta}$ and $x_{\delta}$ be the discrete Euler-Maruyama approximation and the corresponding exact solution of (1) for some maximum step size $\delta>0$ starting at time $t_{0}, X_{0}^{\delta}$ and $x_{0}^{\delta}$ respectively. We shall say that the Euler-Maruyama approximation is numerically stable if for any finite interval $\left[t_{0}, T\right]$ there exist a positive constant $\Delta_{0}$ such that for each $\epsilon>0$ and each $\delta \in\left(0, \Delta_{0}\right)$ the following condition holds

$$
\lim _{\left|X_{0}^{\delta}-x_{0}^{\delta}\right| \rightarrow 0}\left[\sup _{t_{0} \leq t \leq T} P\left(\left|X_{t}^{\delta}-x_{t}^{\delta}\right| \geq \epsilon\right)\right]=0
$$

Proof. Assuming Lipschitz and linear growth conditions and in addition

$$
\sup _{t_{0} \leq t \leq T} E\left(|x(t)|^{2}\right)<\infty
$$

we first consider equation (1) on $t \in\left[\tau_{0}, \tau_{1}\right]$. Now, according with Theorem 4.1 in $([15])$ applied to this interval, we have the following result

$$
Z(t)=\sup _{0 \leq s \leq t} E\left[\left|X_{s}^{\delta}-x_{s}^{\delta}\right|^{2}\right] \leq\left|X_{0}^{\delta}-x_{0}^{\delta}\right|^{2}+K \int_{0}^{t} Z(s) d s
$$

by virtue of the Gronwall inequality

$$
\sup _{0 \leq s \leq t} E\left[\left|X_{s}^{\delta}-x_{s}^{\delta}\right|^{2}\right] \leq \exp \int_{0}^{t} d s\left|X_{0}^{\delta}-x_{0}^{\delta}\right|^{2}
$$


by virtue of the Chevyshev Inequality, the above expression is equivalent to

$$
P\left(\left|X_{t}^{\delta}-x_{t}^{\delta}\right|^{2} \geq \epsilon\right) \leq \frac{1}{\epsilon}\left|X_{0}^{\delta}-x_{0}^{\delta}\right|^{2} t
$$

by applying the corresponding limit

$$
\lim _{\left|X_{0}^{\delta}-x_{0}^{\delta}\right| \rightarrow 0}\left[\sup _{t_{0} \leq t \leq T} P\left(\left|X_{t}^{\delta}-x_{t}^{\delta}\right|^{2} \geq \epsilon\right)\right] \leq \lim _{\left|X_{0}^{\delta}-x_{0}^{\delta}\right| \rightarrow 0} \frac{1}{\epsilon}\left|X_{0}^{\delta}-x_{0}^{\delta}\right|^{2} t=0
$$

then

$$
\lim _{\left|X_{0}^{\delta}-x_{0}^{\delta}\right| \rightarrow 0}\left[\sup _{t_{0} \leq t \leq T} P\left(\left|X_{t}^{\delta}-x_{t}^{\delta}\right|^{2} \geq \epsilon\right)\right]=0
$$

in consequence

$$
\lim _{\left|X_{0}^{\delta}-x_{0}^{\delta}\right| \rightarrow 0}\left[\sup _{t_{0} \leq t \leq T} P\left(\left|X_{t}^{\delta}-x_{t}^{\delta}\right| \geq \epsilon\right)\right]=0
$$

the next step is to apply the same procedure to the interval $t \in\left[\tau_{1}, \tau_{2}\right]$ and so on, up to the interval $t \in\left[\tau_{k+1}, T\right]$. At this point the proof is completed by ensuring stability en each interval determined by the evolution of the Markov chain

Remark 3 The numerical stability criterion applies only to step sizes $\delta>0$ that are less than some critical value $\Delta_{0}$, which will usually depends on the interval $\left[t_{0}, T\right]$, and the specific problem that we attempt to solve.

\subsection{Numerical experiments}

In this part of the paper we show some properties of our numerical approach under the scope of numerical simulations.

Let $w_{t}$ be an scalar Brownian motion. Let $r(t)$ be a right-continuous Markov chain taking values in $S=\{1,2\}$ with the generator

$$
\Gamma=\left(\gamma_{i j}\right)_{2 \times 2}=\left[\begin{array}{cc}
-1 & 1 \\
\gamma & -\gamma
\end{array}\right]
$$

where $w_{t}$ and $r(t)$ are assumed to be independent.

We consider the one-dimensional stochastic differential equation with Markovian switchings

$$
d x(t)=a(r(t)) x(t) d t+b(r(t)) x(t) d B_{t}
$$

that evolves in the finite time interval $[0,1]$, where

$$
a(1)=1, a(2)=2 ; b(1)=2, b(2)=1 .
$$

The equation (13) can be regarded as a set of two equations as follows

$$
\begin{aligned}
& d x(t)=x(t) d t+2 x(t) d B_{t} \\
& d x(t)=2 x(t) d t+x(t) d B_{t}
\end{aligned}
$$

switching from one to the other according with the evolution of the Markov chain $r(t)$. 


\subsubsection{Results}

The first exercise consists on simulate the exact solution of (13), the approximate solution based on the EM-method proposed by Yuao and Mao in [16] and to compare them with our method. Figure 1 shows one sample path of the process (13) by using the exact solution, the Yuan and Mao approximation and our method. The top panel illustrates the evolution of the geometric Brownian motion with switchings based on the exact solution (continuos blue line) and by using the Yuan and Mao approximation (dashed red line) ) for a stepsize $\Delta t=2^{-8}$. As we can observe their numerical approximation diverges from the exact solution. The low panel shows the approximation of the EM-based method proposed in this paper with respect to the exact solution. It is clear that our method provides a so much better approximation to the exact solution than the original method above cited (see upper panel). Nowadays, to verify that this fact is more than a casualty we implement a second exercise. In this exercise we offer a numerical test about strong convergence error between the alternative methods of solution (numerical solutions). In this numerical test, we focus on the endpoint $t=T$. We consider "the strong error measure" defined by

$$
e_{\Delta t}^{\text {strong }}=E\left(\left|x(T)-X_{L}^{\Delta}\right|\right), \text { where } L \Delta t=T
$$

which evaluates the EM endpoint error in the strong sense (see [9]). We compute 1000 discretized Brownian paths over $[0,1]$, with $\delta t=2^{-10}$. For each path, the EM-approximation is computed with 5 different stpesizes: $\Delta t=2^{p-1} \delta t$ for $1 \leq p \leq 5$. The endpoint error in the sth sample path is stored and after all the computations the mean value is calculated. According to Theorem 2 the inequality

$$
e_{\Delta t}^{\text {strong }} \leq C \Delta t^{1 / 2}
$$

holds. So, taking logs on basis 2 , for the approximate equality in (17) we have $\log _{2} e_{\Delta t}^{\text {strong }} \approx \log _{2} C+\frac{1}{2} \log _{2} \Delta t$, then we plots our approximation to $e_{\Delta t}^{\text {strong }}$ against $\Delta t$ on a log-log scale. This procedure yields the strong convergence test in Figure 2 and Figure 3. Simultaneously we have tested different values of $\gamma$ related to the rate of transition (in the transition matrix) of the Markov chain in order to shows the none dependence of the rate of convergence with respect to this parameter.

Figure 2 shows strong convergence test for the Yuan and Mao approach. The right upper panel corresponds to $\gamma=0.5$, the left upper panel correspond to $\gamma=1.5$. From this Figure we appreciate clearly that the method diverges. Figure 3 shows the strong convergence test for our method. The left upper panel corresponds to $\gamma=0.5$; the right upper panel corresponds to $\gamma=1.5$. It is clear from this Figure that our method converges with the order rate anticipated in Theorem 2. This is an important results, because the order of convergence of the EM-method in the $S D E$ area, is preserved in the controlled SDEMS models, so it is independent of the gamma values. 

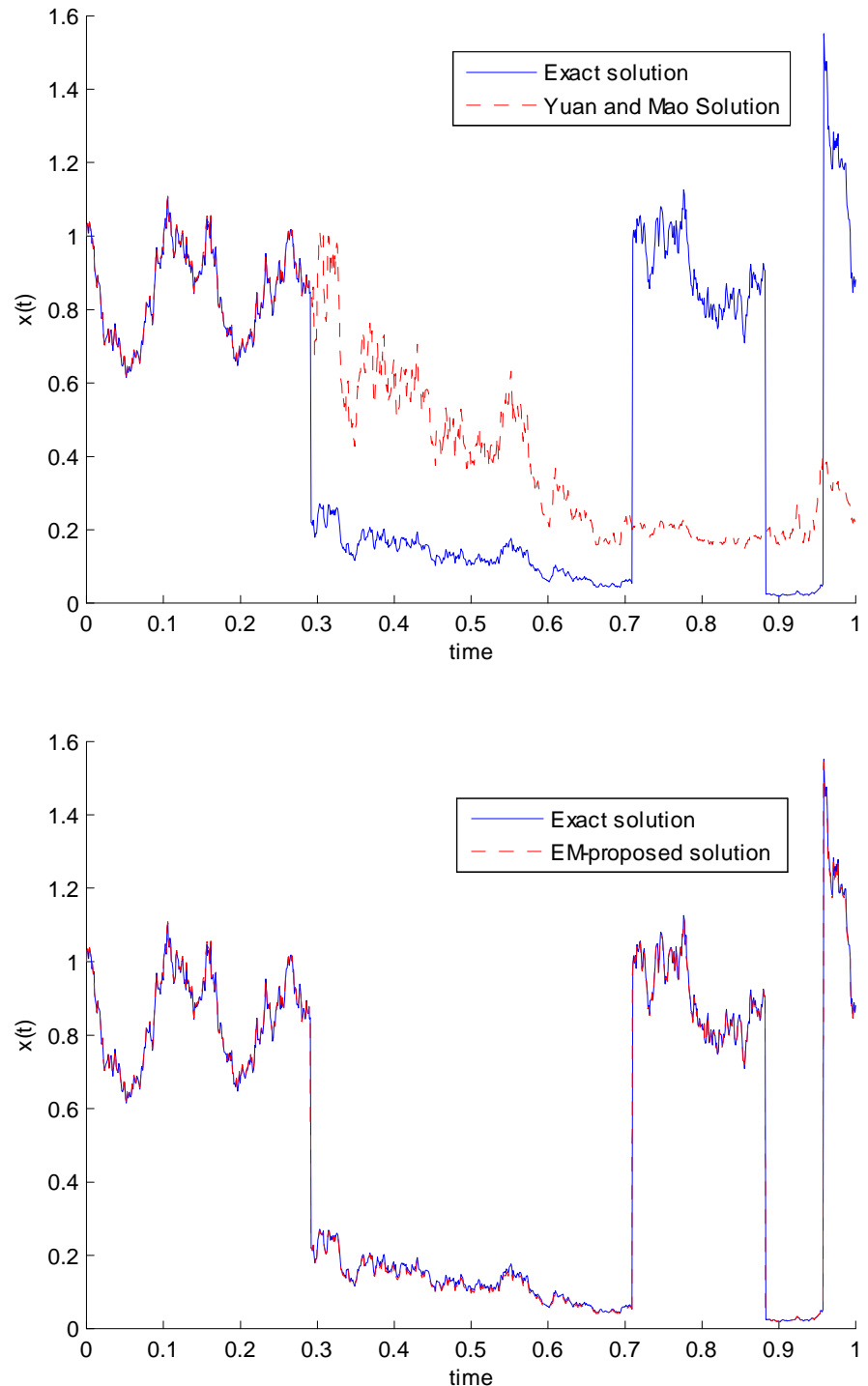

Figure1. Comparison of the exact and the approximated solutions of the geometric Brownian motion with Markovian switchings (13) 

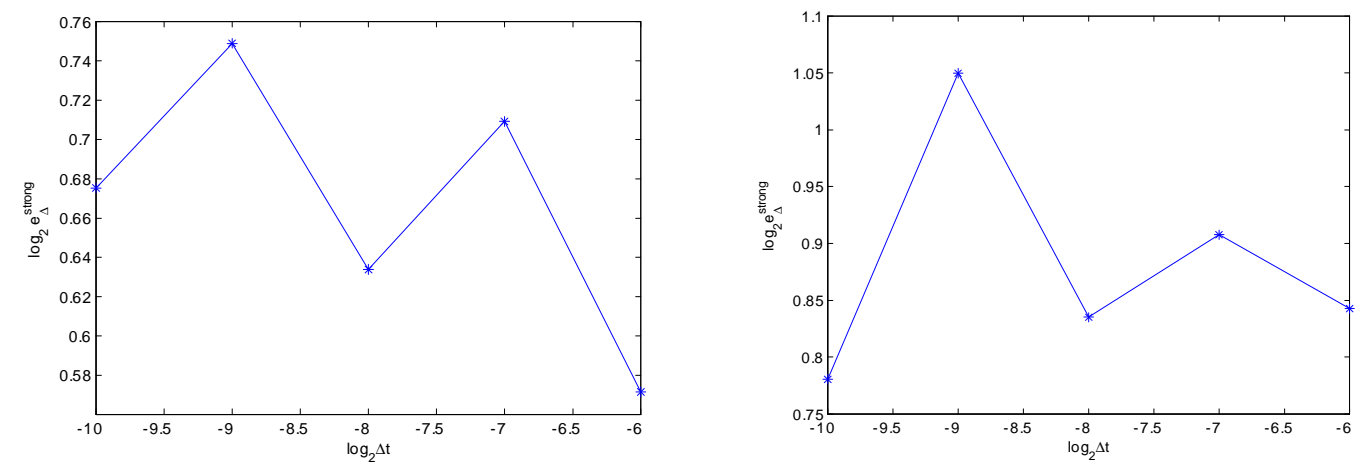

Figure 2. Strong convergence test for the Yuan and Mao method proposed in [16].
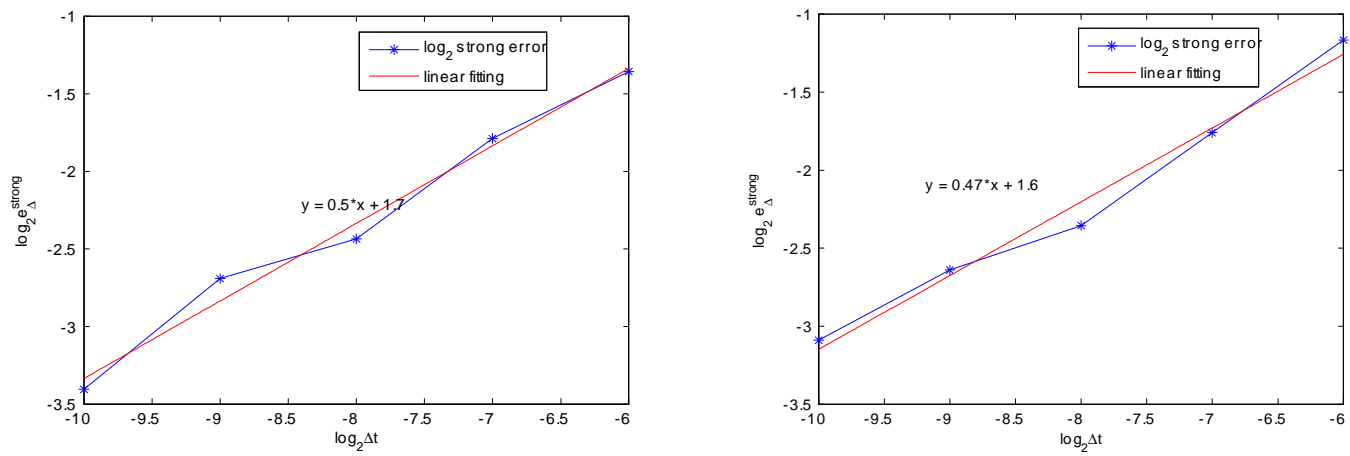

Figure 3. Strong convergence test for the EM-based method proposed in this paper.

\section{Applications}

In order to show the utility of the controlled SDEMS for modelling noisy engineering systems, we have selected an application from the biochemical engineering area. Two scenarios has been chosen for this purpose. In both cases the application is concerned with a feedbatch biochemical reactor. These systems have a widely range of applications in the biochemical engineering area, specially in industrial processes. For example, they are used for producing fuels, pharmaceutical products, foods, among others products, but also they are used for wastewater treatment.

In the first scenario we model the production of penicillin in a bioreactor under a noisy environment that includes changes in structural parameters related to reaction kinetics. In the second scenario we model a bioreactor used to wastewater treatment. In the last case we attempt to show the effects of a noisy environment that includes abrupt changes in input parameters (flows and concentrations) as is common during the operation of these systems. Even tough the industrial scales of the problems are different, the underlying principles that 
govern the behavior of the bioreactor are the same. We emphasize that these systems are very challenging from the control point of view, so it is extremely important to study its behavior under a noisy environments. The values of the parameters used in both applications has been taken from [19].

\subsection{Biochemical reactors (brief description)}

A biochemical feedbatch reactor is an engineering systems where a chemical transformation is carry out by biological entities. Briefly, these equipments consist of : (i) A mechanical vessel, that is a recipe where the biochemical reactions are carried out by microorganisms that are previously cultivated. (ii) The biological material that is transformed via specific reactions. (iii) An input consisting on a flux with nutrients and water meanly, that are added continuously to the vessel during the system operation.

\subsection{Scenario 1}

We model a bioreactor for producing penicillin. The system is described as follows

- State variables. $x_{1}$ : concentration of biomass (biological material), $x_{2}$ : concentration of substrate (nutrients), $x_{3}$ : concentration of product (penicillin) and $x_{4}:$ Volume of reaction

- Input Parameters. F : Feed flow rate and $C$ : Inlet substrate concentration.

- Structural parameters. $\left(\mu_{m}, K_{m}, K_{i}\right.$ and $\left.\nu\right)$ : kinetic parameters; $\left(Y_{x_{1}}\right.$ and $\left.Y_{x_{3}}\right)$ : yield coefficients.

Scientific basis: the pyshical interrelations between state variables is based on the mass and energy balance equations.

Source of noise: we assume multiplicative noise in $x_{1}$ and $x_{3}$ as a product of low concentrations and high sensibility between species in these type of systems, additive noise is considered in $x_{2}$ in order to model smooth and unpredictable environmental fluctuations such as temperature, $\mathrm{pH}$, among others. In addition we model structural changes related to the biological activity of the microorganisms concentrated in the kinetic parameter $\mu_{m}$. We assume that this parameter could take different values from a finite fixed set according with the evolution of a Markov chain.

Control protocol: In this system the control is given by the feed flow rate $F$.

Operation time: The time operation is equivalent to eight hours. 
Dynamics: the bioreactor dynamics is modeled as a controlled SDEMS as follows

$$
\begin{aligned}
& d x_{1}(t)=\left(\frac{r(t)}{K_{m}+x_{2}} x_{2}(t) x_{1}(t)-\frac{F}{x_{4}} x_{1}(t)\right) d t+\sigma_{1} x_{1}(t) d w_{1} \\
& d x_{2}(t)=\left[\left(\frac{r(t)}{K_{m}+x_{2}(t)} \frac{1}{Y_{1}} x_{2}(t) x_{1}(t)+\frac{v}{Y_{3}} x_{1}(t)\right)+\frac{F}{x_{4}(t)}\left(C-x_{2}(t)\right)\right]+\sigma_{2} d w_{2} \\
& d x_{3}(t)=\left(v x_{1}(t)-\frac{F}{x_{4}(t)} x_{3}(t)\right) d t+\sigma_{3} x_{3}(t) d w_{3} \\
& d x_{4}(t)=F
\end{aligned}
$$

The dynamics of the system drives a four-dimensional state variable $x(t) \in$ $\mathbb{R}_{+}^{4}$, and it is influenced by a three-dimensional increment of the brownian motion $d w \in \mathbb{R}^{3}$. The drift function $f: \mathbb{R}_{+}^{4} \times U \times S \rightarrow \mathbb{R}_{+}^{4}$ and the diffusion function $g: \mathbb{R}^{4 \times 3} \rightarrow \mathbb{R}_{+}^{4}$ complete the description of he system. In a matricial way, the elements of system (1) are summarized as follows

$$
\begin{aligned}
& x(t)=\left(x_{1}(t), x_{2}(t), x_{3}(t), x_{4}(t)\right)^{T} \\
& d w=\left(d w_{1}, d w_{2}, d w_{3}\right)^{T} \\
& f=\left[\begin{array}{c}
\left(\frac{r(t)}{K_{m}+x_{2}} x_{2}(t) x_{1}(t)-\frac{F}{x_{4}} x_{1}(t)\right) \\
{\left[\left(\frac{r(t)}{K_{m}+x_{2}(t)} \frac{1}{Y_{1}} x_{2}(t) x_{1}(t)+\frac{v}{Y_{3}} x_{1}(t)\right)+\frac{F}{x_{4}(t)}\left(C-x_{2}(t)\right)\right.}
\end{array}\right] \\
& g=\left[\begin{array}{ccc}
\sigma_{1} x_{1} & 0 & 0 \\
0 & \sigma_{2} & 0 \\
0 & 0 & \sigma_{3} x_{3} \\
0 & 0 & 0
\end{array}\right]: \mathbb{R}^{4 \times 3} \rightarrow \mathbb{R}_{+}^{4}
\end{aligned}
$$

The input parameters affine to the system are $F$ and $S$, where

$$
u=F \in \mathbb{R}_{+}
$$

Observe that the Markov chain $r(t)$ is related to the structural parameter $\mu_{m}$, which measure the rate of growth of the microorganisms. In this example we consider a two state Markov chain. We assume that the structural parameter will take the values $\mu_{m}(1)=0.53$ and $\mu_{m}(2)=0.23$ depending on the state of the underlying Markov chain.

On the other hand, the vector parameter $\sigma=\left(\sigma_{1}, \sigma_{2}, \sigma_{3}\right)$ is related to the intensity of the additive and multiplicative noise according with (22)-(25). Due to the absence of noise in the state variable $x_{4}$ (the volume of the bioreactor), we have just a three dimensional increment of the brownian motion. In other words, the dynamics of the volume of the bioreactor, determined by the feed flow rate $F$ (control protocol), is assumed constant. In this case $F=0.5 \frac{\mathrm{lit} \text {. }}{\mathrm{hr} \text {. }}$ 
Notice that in this modeling application we fix the behavior of the Markov chain according to some design values. In practical applications the transition states of the Markov chain should be estimated probably on line.

\subsubsection{Lipschitz conditions}

In order to apply our model approach it is necessary to ensure that functions $f$ and $g$ in (24)-(25) verify the Lipschitz conditions. Fortunately these functions are smooth enough to guarantee a well defined solution as is shown in preliminary studies in [4].

\subsubsection{Numerical Simulation}

Our EM-method has been implemented according to the procedure described in Section 2, with a stepsize $\Delta t=2^{-9}$. The integration is carried out over the finite interval $[0, T]$ with $T=8$. Table 1 provides the numerical values of structural and input parameters. Table 2 we show the values of the initial conditions, also Table 3 presents the noise intensity parameters $\sigma$ considered.

\begin{tabular}{|c|c|c|}
\hline Parameter & Value & units \\
\hline$K_{m}$ & 1.2 & $\frac{g}{\text { lit. }}$ \\
\hline$K_{i}$ & 22 & $\frac{g}{\text { lit. }}$ \\
\hline$Y_{1}$ & 0.4 & none \\
\hline$Y_{3}$ & 1 & none \\
\hline$v$ & 0.5 & $\frac{\text { lit. }}{\text { hrs. }}$ \\
\hline$S$ & 20 & $\frac{\text { lit. }}{\text { hrs. }}$ \\
\hline$F$ & 0.5 & $\frac{\text { lit. }}{\text { hrs. }}$ \\
\hline
\end{tabular}

Table1.

\begin{tabular}{|c|c|c|}
\hline State variable & value & units \\
\hline$x_{1}(0)$ & 1 & $\frac{g}{\text { lit. }}$ \\
\hline$x_{2}(0)$ & 0 & $\frac{g}{\text { lit. }}$ \\
\hline$x_{3}(0)$ & 0 & $\frac{g}{\text { lit. }}$ \\
\hline$x_{4}(0)$ & 2 & lit. \\
\hline
\end{tabular}

Table 2 .

\begin{tabular}{|c|c|}
\hline Parameter & value \\
\hline$\sigma_{1}$ & 0.05 \\
\hline$\sigma_{2}$ & 0.05 \\
\hline$\sigma_{3}$ & 0.05 \\
\hline
\end{tabular}

Table 3 . 
Markov chain simulation: let $r(t)$ be a right-continuous Markov chain taking values in $S=\{1,2\}$ with the generator.

$$
\Gamma=\left(\gamma_{i j}\right)_{2 \times 2}=\left[\begin{array}{cc}
-1 & 1 \\
\gamma & -\gamma
\end{array}\right]=\left[\begin{array}{cc}
-1 & 1 \\
0.5 & -0.5
\end{array}\right]
$$

The one-step transition probability matrix considered is given by

$$
P(\Delta)=\left(P_{i j}(\Delta)\right)_{2 \times 2}=e^{\Delta \Gamma}=\left[\begin{array}{ll}
0.9980 & 0.0020 \\
0.0010 & 0.9990
\end{array}\right]
$$

which is determined by the discrete Markov chain $r_{k}=r(k \Delta)$, with $\Delta=2^{-9}$ (the same value as the stepsize of integration).

Results Figure 1 shows the profiles of the state variables related to the deterministic dynamics of the system (22)-(25). The idea is to have a point of reference to compare the noisy scenarios with the deterministic case. The left upper panel shows the biomass concentration dynamics, which is essential for the production of the final product (penicillin). The right upper panel shows the nutrients concentration dynamics. We observe a clear non-monotonic behavior of this element. The lower panel shows the penicillin concentration dynamics. Notice that the dynamics of the bioreactor volume is not included because it has been considered fixed in this scenario.
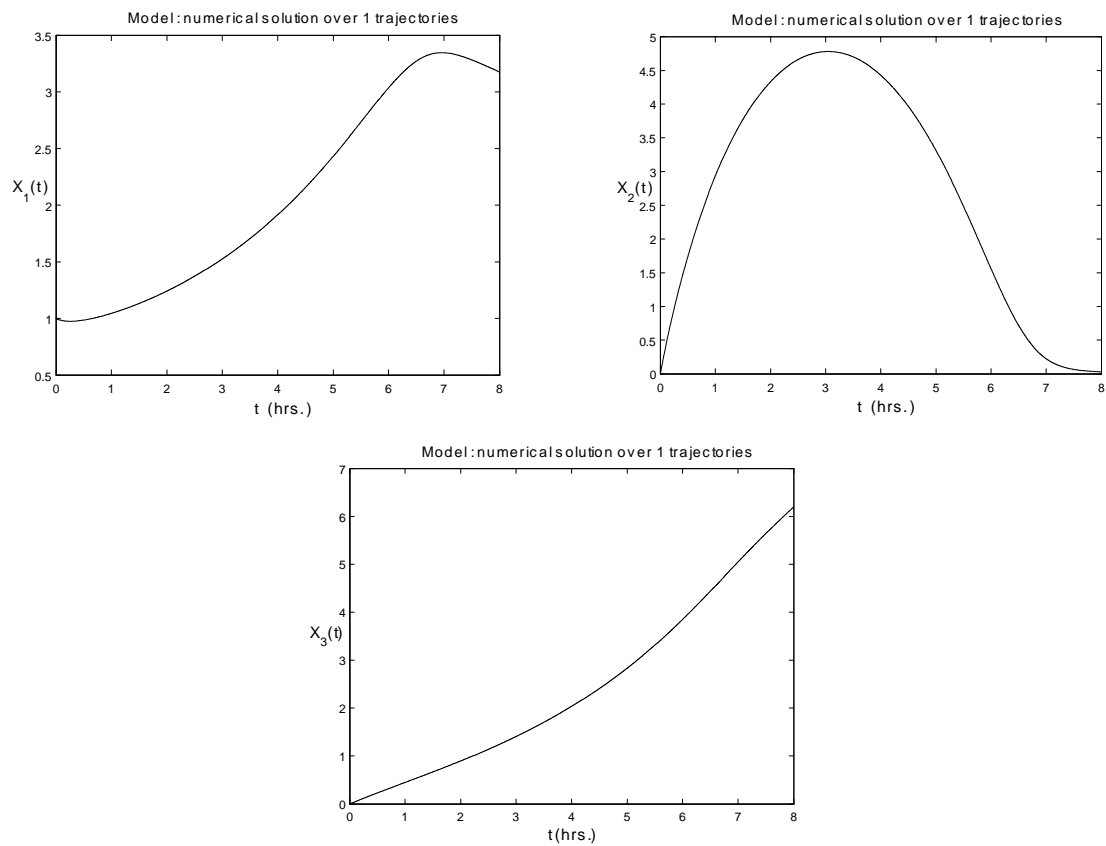

Fig1. State trajectories obtained with the deterministic model. 

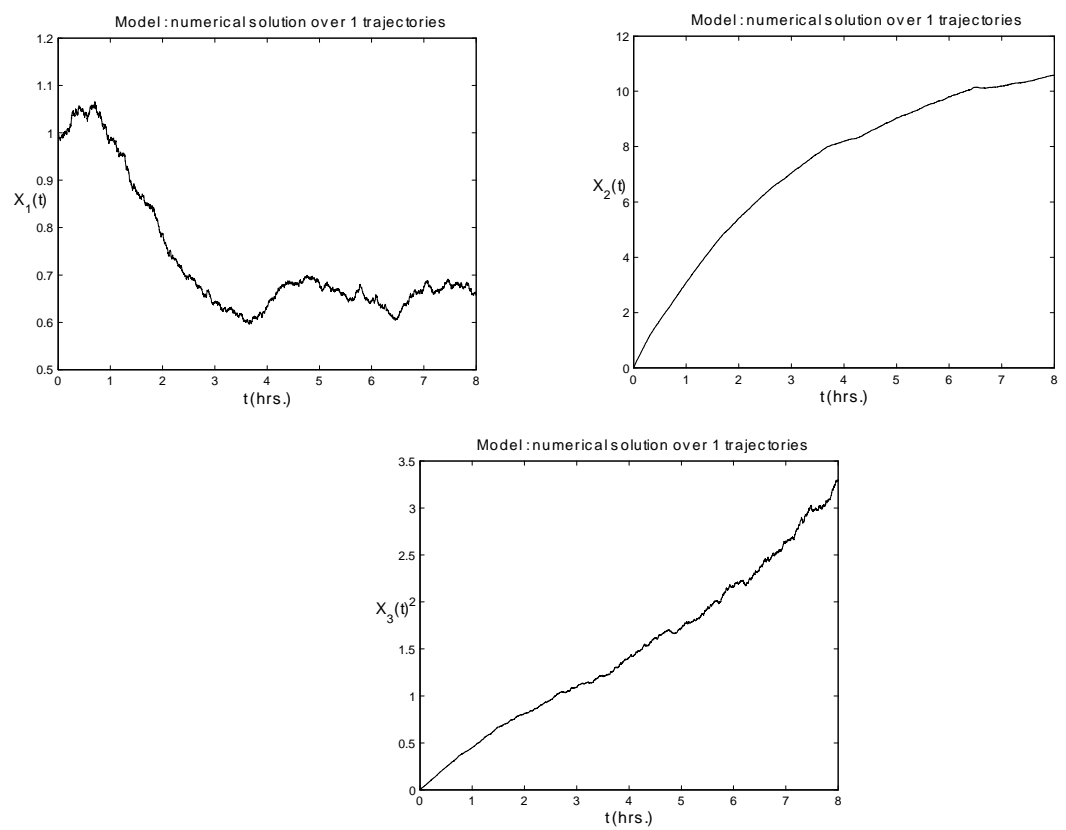

Figure 2. One realization of the state trajectories obtained with the model (22)-(25).

Figure 2 shows the dynamics of the bioreactor under the effects of structural noise modeled as in (22)-(25). The left upper panel shows the biomass concentration dynamics under the noisy environment induced. The profile shows the existence of at least two regimes in the dynamics of this state variable. The right upper panel shows the substrate concentration dynamics. We appreciate the effects of the structural noise induced by changes in the kinetic parameter. It is clear that the structural noise induces a decrease in the biological activity. Then the substrate is not profited suitably. Therefore, the concentration of the final product decreases as is showed in the lower panel. Of course we refer to the quantitative level. In this case the maximum value is about $2.5 \frac{g r}{l i t}$ instead to $6.5 \frac{g r}{l i t}$ as shows the Figure 1 (lower panel).

Summarizing, the simulated effects of additive, multiplicative and structural noise have serious effects in the biological activity of the bioreactor as usually occur in the reality. In industrial scenarios this kind of variability has tremendous impact in cost and efficiency of the processes. Strategies of control that take into account these scenarios are necessaries to take better decisions about the operation and yielding of these equipments.

\subsection{Scenario 2}

We model a feedbatch bioreactor used in waste-water treatment. In this case the bioreactor is used for treating organic contaminants in industrial effluents. Abrupt fluctuations arrives to this equipment as a product of changes in the concentration of the organic material that is treated. The control action is 
to increase or decrease the feed flow-rate to face up to the variations on the concentration above commented. We are not assuming an optimal strategy, but in a second step it could be solved from the optimal control perspective. We describe this system as follows

- State variables. $x_{1}$ : concentration of biomass, $x_{2}$ : concentration of substrate (residues), $x_{3}$ : concentration of water treated and $x_{4}$ : Volume of reaction.

- Input Parameters. F : Feed flow rate and $C$ : Inlet substrate concentration.

- Structural parameters. $\left(\mu_{m}, K_{m}, K_{i}\right.$ and $\left.\nu\right)$ : kinetic parameters; $\left(Y_{x_{1}}\right.$ and $\left.Y_{x_{3}}\right)$ : yield coefficients.

Scientific basis: pyshical interrelations between state variables is based on the balance equations for mass and energy.

Source of noise: we assume additive noise in all the state variables because the system works at high levels of concentrations. The additive noise is considered to model smooth and unpredictable environmental fluctuations such as temperature, $\mathrm{pH}$, among others. In addition, abrupt fluctuation are induced through exogenous and control variables.

Control protocol: in this system the control is given by the feed flow rate F.

Operation time: eight hours.

Dynamics: the dynamics of the bioreactor is modeled as a controlled SDEMS. We detail the model as follows

We have a four-dimensional state variable $x(t) \in \mathbb{R}_{+}^{4}$, and a four-dimensional increment of the brownian motion $d w \in \mathbb{R}^{3}$. The drift and the diffusion functions are $f: \mathbb{R}_{+}^{4} \times U \times S \rightarrow \mathbb{R}_{+}^{4}$ and $g: \mathbb{R}^{4 \times 4} \rightarrow \mathbb{R}_{+}^{4}$ respectively. The dynamics of the systems is described as follows

$$
\begin{aligned}
x(t)= & \left(x_{1}(t), x_{2}(t), x_{3}(t), x_{4}(t)\right)^{T} \\
d w= & \left(d w_{1}, d w_{2}, d w_{3}, d w_{4}\right)^{T} \\
f= & \left(\frac{\mu_{m}}{K_{m}+x_{2}} x_{2}(t) x_{1}(t)-\frac{F(t)}{x_{4}} x_{1}(t)\right) \\
& {\left[\begin{array}{c}
\left.\left(\frac{\mu_{m}}{K_{m}+x_{2}(t)} \frac{1}{Y_{1}} x_{2}(t) x_{1}(t)+\frac{v}{Y_{3}} x_{1}(t)\right)+\frac{F(t)}{x_{4}(t)}\left(C(t)-x_{2}(t)\right)\right] \\
\left(v x_{1}(t)-\frac{F(t)}{x_{4}(t)} x_{3}(t)\right) \\
F(t)
\end{array}\right] } \\
g= & {\left[\begin{array}{cccc}
\sigma_{1} & 0 & 0 & 0 \\
0 & \sigma_{2} & 0 & 0 \\
0 & 0 & \sigma_{3} & 0 \\
0 & 0 & 0 & \sigma_{4}
\end{array}\right] }
\end{aligned}
$$

The control protocol is $u(C(t))=F(t)$, where $C(t)$ evolves as a two state Markov chain. Notice that it is an ideal assumption that suggest an immediate 
response of the controller to the abrupt changes. So we have that $C(1)=20 \frac{g}{\text { lit. }}$, $C(2)=30 \frac{g}{l i t .}$ and $F(1)=0.53 \frac{\text { lit. }}{h r .}, F(2)=0.053 \frac{\text { lit. }}{h r .}$.

The noise intensity parameters considered correspond to the four-dimensional parameter $\sigma=\left(\sigma_{1}, \sigma_{2}, \sigma_{3}, \sigma_{4}\right)$ related with the additive noise previously described. The same level of noise intensity is assumed for all the state variables (see Table 4).

\subsubsection{Lipschitzian conditions}

From [4] we conclude that functions $f$ and $g$ exhibit enough regularity properties to provide a well defined solution and a well suitable numerical approach.

\subsubsection{Numerical simulation}

For this scenario we have used the same technical consideration concerned to the simulation details in the Scenario 1. The values of the structural parameters and initial conditions remains unchanged. Table 4 shows the values of the noise intensity parameter $\sigma$.

\begin{tabular}{|c|c|}
\hline Parameter & Value \\
\hline$\sigma_{1}$ & 0.05 \\
\hline$\sigma_{2}$ & 0.05 \\
\hline$\sigma_{3}$ & 0.05 \\
\hline$\sigma_{4}$ & 0.1 \\
\hline
\end{tabular}

Table 4.

Results We summarize the results of the simulation of the Scenario 2 in Figure 3 . 

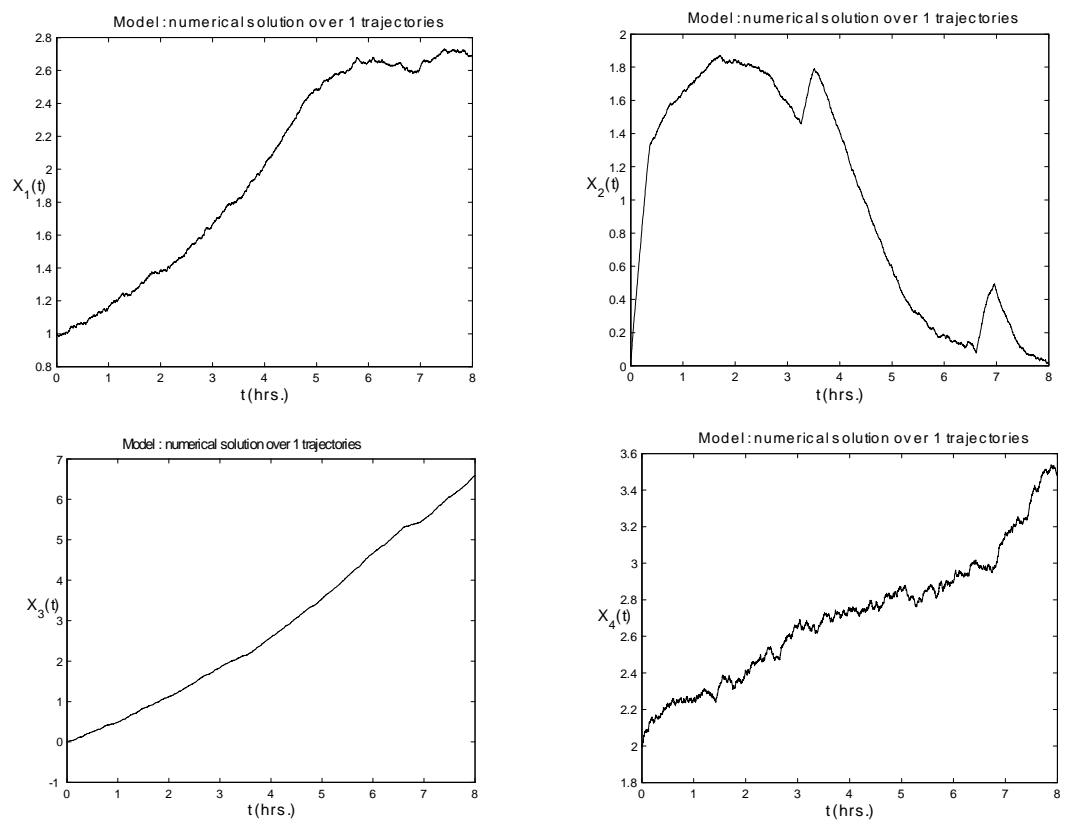

Figure 3. One realization of the state trajectories obtained with the model (26)-(29)

The left upper panel shows one trajectory (realization) of the stochastic process related to the biomass concentration dynamics. The right upper panel shows one trajectory of the stochastic process related to the substrate concentration dynamics followed during the operation of the bioreactor. The left lower panel shows one trajectory (realization) of the stochastic process related to the dynamics followed by the water treated concentration dynamics. The right lower panel shows one realization of the stochastic process related to the dynamics of the volume of the bioreactor.

It is clear that the system dynamics (26)-(29) reflects the effects of the noise induced by the model in the bioreactor. However, in this case we observe that the effects are far from ones exhibited in Figure 2. The first profile corresponds to the biomass concentration, which is directly related to biological activity in the bioreactor. We note that this activity don't reflect any structural change related with to the presence of regimes. The explanation of this fact is attributed to the absence of structural noise in the system, this affirmation is supported by the dynamics of the substrate (level of nutrients). We observe that its behavior is very similar to the deterministic case, even though we appreciate abrupt disturbances in its behavior during the operation of the bioreactor. The effects of the noise in the dynamics of the water treated are negligible in this case, this fact is reasonable given that there is not structural variations in the biological activity but also due to the control strategy. In other words, the effects of additive and input parametric noise in macro-scale systems as a waste water bioreactor are non relevant if they are not connected in some way with structural 
changes in the dynamics of the microorganisms and if the control action are sufficient fast and right.

\section{Conclusions}

We provide a systematic treatment for engineering dynamical systems under intrinsic and external noisy environments based on controlled SDEMS models. This treatment allows to describe, from a modelling point of view, controlled engineering dynamical systems in more realistic scenarios. The idea is to show the flexibility of these models for capturing the effects of structural or abrupt changes in the behavior of engineering systems .

The methodology here proposed does not substitute other ones based on measure and estimation to treat noise, however represents a valuable and promising complementary tool based on stochastic simulation, in the direction of gain insight in this kind of phenomena.

Elementary applications in biochemical processes illustrate the different role that play structural parameters and input parameters in the dynamics of the system when this is affected or is part of the noisy environment itself.

Two relevant aspects are considered in this work. The first one from a theoretical point of view and the second one is related to practical issues. In the former, existence and uniqueness conditions for the mathematical solution of the model are established. In the second one a numerical scheme based on the EM- method is proposed to provide practical solutions (numerical solutions) to the controlled SDEMS equations. In the theoretical context, Lipschitz conditions are very restrictive for modeling medium and high nonlinear complex engineering systems. Then, further research in this area should be developed in order to relax that conditions. With respect to the numerical approximation, we guarantee that the EM-scheme verify the convergence and stability properties which are necessaries in order to have right approximations to the real solutions (analytical solutions). The EM method is an ad hoc method to develop numerical approximations to the $S D E M S$ models, easy to implement and fast, but in order to treat a more widely spectrum of nonlinear problems we need to develop more efficient and accurate schemes. These methods must also guarantee positivity conditions because we face problems where the state variables represent physical variables that admit just positive values (i.e. Temperatures, concentrations, pressures, etc.). It is very important to consider a more careful treatment for the initial conditions in each regime. In practice we don't know exactly that conditions. So, the numerical approach may depends strongly of this information.

Two main lines-research shall be followed in short and medium time. The first one must be devoted to develop and implement a battery of well defined numerical schemes, to face a widely type of nonlinear problems going from weak to the high nonlinear level. Due to the complexity of the problems we treat, does not exist "the best method". Numerical methods must to be adapted according with the problem is faced up, so it is adviceable to have a battery of methods 
that can be used.

Second line will be devoted to implement optimal strategies of control over these systems according to some efficiency or optimally criteria. We want to develop a practical algorithm to get optimal protocols in engineering noisy systems. Promising techniques based on iterative dynamic programming will be explored.

\section{References}

[1] Arnold, L., 1974. Stochastic Differential Equations: Theory and Applications. Jhon Wiley and Sons.

[2] Arnold, L., 1988. Random Dynamical Systems, Springer-Verlag.

[3] Buffington, J. and Butcher, J.C., 2002. American options with regime switchings, International Journal of Theoretical and Applied Finance, 5, pp. $497-514$.

[4] Cañada, H. and Romera, R.,2008. Diffusion Process with Markovian Switching Applied to Biochemical Processes, Proceedings of International Workshop on Applied Probability (IWAP 2008), Compiègne, France, July 7-10, 2008.

[5] Costa, O.L.V. and Boukas, K., 1998. Necessary and sufficient conditions for robust stability and stabilizability of continuous-time linear with markovian jumps, J.

[6] Cassandras, G. and Lygeros, J., 2006. Stochastic Hybrid Systems: Recent Developments and Research Trends. In:CRC Press, Hybrid Systems for Biochemical Network Modeling and Analysis (HYGEIA)., 1-26.

[7] Gardiner, C. W., 1983. Handbook of stochastic methods for Physies, Chemistry and Natural Sciences. Springer.

[8] Ghosh, M. K., Arapostahis, A. and Marcus, S. I., 1993. Optimal control of switching diffusions with applications to flexible manufacturing systems, SIAM Journal of Control and Optimization, 31, pp. 1183-1204.

[9] Higham, D.J., 2001. An Algorithmic Introduction to Numerical Simulation of Stochastic Differential Equations. SIAM., 43, 525-546.

[10] Higham, D.J., Mao, X., 2005. Strong convergence of Euler-type methods for nonlinear stochastic differential equations. SIAM Journal on Numerical Analysis, 40, 3, pp., 1041-1063.

[11] Karatzas, I. and Shreve, S.E., 1988. Brownian Motion and Stochastic Calculus. Springer. 
[12] Kloeden, P.E., Platen, E., 1992. Numerical Solutions of Stochastic Differential Equations. Springer-Verlag, Berlin, New York.

[13] Kloeden, Peter, E., Platen, E. and Shurz, H., 2003. Numerical Solution of SDE Through Computer Experiments. Springer.

[14] Lobry, J.R., Flandrois, G.C. and Pave, A., 1992. Monod's Bacterial Growth Model Revisited. Bulletin of Mathematical Biology, 54, 117-122.

[15] Mao, X. and Yuan, CH., 2006. Stochastic Differential Equations with Markovian Switchings, Imperial College Press, London.

[16] Mao, X. and Yuan, CH., 2004. Convergence of the Euler Maruyama Method for Stochastic Differential Equations with Markovian Switchings. Mathematics and Computers in Simulations, 64, 223-235.

[17] Monod, J., 1949. The grow of Bacterial cultures. Rev. Microbiology, 3, 371-394.

[18] Øksendal, B.,1995. Stochastic Differential Equations: An introduction with applications. Sth. Ed., Springer..

[19] Srinivasan, B. et al., 2003. Dynamic Optimization of Batch Processes I: Characterization of the nominal solutions. Computer and Chemical Engineering, 29, 1-26.

[20] Turner, T.E., Schnell S. and Burrage, K., 2004. Stochastic approaches for modeling in vivo reactions analysis. Computational Biology and Chemistry, 28, 165-178. Optimization Theory Appl., 99, pp., 1155-1167.

[21] Wong, E., and Hajek, B., 1985. Stochastic Processes in Engineering Systems. Springer. 